\title{
NARRATIVAS VACUNALES Y POLÍTICAS DE VACUNACIÓN CONTRA LA POLIOMIELITIS EN EL CONTEXTO DE LA PROVINCIA DE ALICANTE (1963-1978)
}

\author{
José V. Toledo-Marhuenda \\ Universidad Miguel Hernández \\ Email: josetoledo@umh.es \\ ORCID iD: http://orcid.org/0000-0002-5336-6837 \\ Rosa Ballester \\ Facultad de Medicina. Universidad Miguel Hernández \\ Email: rosa.ballester@umh.es \\ ORCID iD: http://orcid.org/0000-0002-7870-4185
}

Recibido: 8 febrero 2018; Aceptado: 15 mayo 2019

Cómo citar este artículo/Citation: Toledo-Marhuenda, José V.; Ballester, Rosa (2020), “Narrativas vacunales y políticas de vacunación contra la poliomielitis en el contexto de la provincia de Alicante (1963-1978)", Asclepio 72 (1):p294. https://doi.org/10.3989/ asclepio.2020.03.

RESUMEN: El análisis a pequeña escala es una vía de abordaje historiográfico que abrió, en su momento, nuevos caminos en la investigación. La proximidad del objeto de estudio, permite profundizar en los determinantes propios y específicos y en las realidades y prácticas, por ejemplo, de las campañas de inmunización masiva contra la poliomielitis diseñada a nivel nacional pero implementadas localmente, como en el estudio de caso que abordamos. Utilizando como modelo el ámbito sanitario provincial de Alicante, el trabajo añade datos para completar la información de proximidad sobre estas campañas y de ese modo, completar una visión de conjunto y un análisis comparado tanto en un ámbito externo como en un ámbito interno interterritorial, nacional y provincial, así como el seguimiento de las directrices dadas por los organismos nacionales e internacionales.

En segundo término, dotar de significado al conjunto de actividades llevadas a cabo por diferentes actores, instituciones y medios de comunicación, implicados en el proceso de implantación, cobertura y seguimiento de las campañas de vacunación contra la polio en el ámbito provincial alicantino. El uso de narrativas de personas afectadas por la enfermedad permite añadir elementos relevantes sobre experiencias personales en el contexto de las campañas vacunales.

PALABRAS CLAVE: poliomielitis; vacunación; narrativas vacunales; Alicante; España; siglo XX.

\section{VACCINE NARRATIVES AND POLIO VACCINATION POLICIES IN THE CONTEXT OF THE PROVINCE OF ALICANTE (1963-1978)}

\begin{abstract}
Small-scale analysis is a path of historiographical approach that opened, at the time, new paths in research. The proximity of the study object allows us to delve into our own and specific determinants and the realities and practices of, for example, mass immunization campaigns against polio, designed at the national level but implemented locally, as in the case study that we discuss. Using as a model the provincial sanitary area of Alicante, the work adds data to complete the proximity information on these campaigns and thus, complete an overview and a comparative analysis both in an external and in an internal interterritorial, national scope and provincial level as well as the follow-up of the guidelines given by the national and international organisms. Secondly, to give meaning to the set of activities carried out by different actors, institutions and media, involved in the process of implementation, coverage and follow-up of polio vaccination campaigns in the province of Alicante. The use of narratives of people affected by the disease allows to add relevant elements about personal experiences in the context of the vaccine campaigns.
\end{abstract}

KEY WORDS: poliomyelitis; vaccination; vaccine narratives; Alicante; Spain; twentieth century.

Copyright: (C) 2020 CSIC. Este es un artículo de acceso abierto distribuido bajo los términos de la licencia de uso y distribución Creative Commons Reconocimiento 4.0 Internacional (CC BY 4.0). 


\section{INTRODUCCIÓN}

La incorporación de los programas poblacionales de vacunación junto a la universalización de la atención médica fueron las dos grandes medidas de mayor impacto sobre la morbilidad y la mortalidad en el último tercio del siglo XX en España.

La presencia de brotes epidémicos de poliomielitis, una patología que tuvo un enorme impacto social y mediático en los países occidentales en el siglo XX (Smallmann-Raynor y Cliff, 2006), por afectar a edades infantiles, por la ausencia de tratamientos eficaces y por las secuelas de las formas paralíticas (Toledo Marhuenda, 2013), experimentó en España un ascenso continuado hasta 1963 , cuando se inició la campaña de inmunización masiva de la población infantil (Rodríguez Sánchez; Seco Calvo, 2009; Martínez Navarro, 2013; Porras Gallo, Ayarzagüena Sanz, De las Heras Salord, Báguena Cervellera, 2013; Nájera Morrondo, 2013). En el esquema organizativo de la sanidad española, las Juntas Provinciales de Sanidad fueron las encargadas de la gestión de las vacunaciones hasta que, en 1978, las delegaciones territoriales del Ministerio de Sanidad asumieron, entre otras, estas funciones que luego pasarían a las consejerías de sanidad de las Comunidades Autónomas.

Pese a la supuesta uniformidad de las políticas de vacunación contra la poliomielitis, emanadas del poder central tras la primera campaña de inmunización masiva de 1963-64, el análisis en profundidad de la realidad de las prácticas en entornos locales permite desvelar semejanzas y diferencias sustantivas entre lugares. Estudios recientes nos están permitiendo conocer de primera mano estas realidades y el papel de los distintos actores implicados. Los contextos locales, como se ha podido comprobar en otros estudios (Porras Gallo y Báguena Cervellera, 2015; Rodríguez Sánchez, 2015) y en los testimonios del grupo de virólogos y epidemiólogos de la Dirección General de Sanidad y del Centro Nacional de Microbiología, Virología e Inmunología sanitaria, encabezado por Florencio Pérez Gallardo (Nájera et al, 1975), fueron determinantes para entender los problemas surgidos en el transcurso de los programas de vacunación, así como las diferencias entre una u otra región.

El primer calendario de vacunación en España se instauró en 1975 con el objetivo de mejorar las coberturas y que las vacunaciones se efectuaran de forma continuada, aunque la vacunación masiva y sistemática contra la poliomielitis comenzó en 1963-64.

Utilizando como modelo el ámbito sanitario provincial de Alicante, la finalidad del trabajo es triple: por un lado, contribuir a completar la información sobre estas campañas, con objeto de obtener una visión de conjunto y un análisis comparado tanto dentro del propio estado español (Porras Gallo, Ayarzagüena Sanz, Delas Heras Salord, Báguena Cervellera, 2013) como fuera de él (Ballester Añón y Porras Gallo, 2012). En segundo término, dotar de significado al conjunto de actividades llevadas a cabo por diferentes actores e instituciones sanitarias del ámbito alicantino, cuyo contexto hemos tenido la ocasión de analizar y que nos sirve de marco de referencia (Ballester Añón, Bernabeu Mestre, Castejón Bolea, Perdiguero Gil, 2012). Finalmente, incorporar los testimonios de personas afectadas por la enfermedad, así como el reflejo en la prensa local de las campañas vacunales.

El estudio comprende desde la década de 1950 hasta el año 1975, fecha que coindice con el fin del régimen franquista y con la implantación del calendario vacunal. La continuidad de esta pauta vacunal fue decisiva en la erradicación de la enfermedad en 1988, con la declaración, en Almería, del último caso de polio en nuestro país.

Las fuentes de datos utilizadas han sido las procedentes de archivos de tipo local y provincial (Archivo Histórico Municipal de Alicante, Archivo de Diputación Provincial de Alicante); datos epidemiológicos, procedentes del Instituto Nacional de Estadística; informes, de organismos internacionales, y reuniones de expertos (OMS) sobre cómo llevar a cabo las campañas y otras estrategias frente a la poliomielitis; publicaciones científicas de epidemiólogos y virólogos de la Escuela Nacional de Sanidad; y la revisión sistemática de todas las noticias que sobre poliomielitis aparecieron en el diario Información con el fin de contrastar la realidad de la práctica de la vacunación local.

La introducción de experiencias personales para reconstruir la realidad de la puesta en marcha de los programas de vacunación en todas sus vertientes, incluida la subjetividad, es un campo de análisis historiográfico poco transitado. A diferencia de la muy estimable tradición de narrativas de enfermedad (Ortiz Gómez et al., 2008), los testimonios que giran en torno a cuestiones de salud pública, como 
en el caso de la población que participó en programas de vacunación, han sido poco explorados por los historiadores debido, en parte, a la dificultad que la identificación y tratamiento de este tipo de fuentes conlleva.

En este trabajo, mediante la técnica de la historia oral, hemos recogido una cincuentena de testimonios de personas que aúnan una triple peculiaridad: residir en el entorno geográfico objeto de estudio; haber vivido en los periodos en los que se pusieron en marcha los programas vacunales $y$, en tercer lugar; padecer la enfermedad y sus secuelas.

Planteado como un estudio de caso, el trabajo se centra en el análisis del seguimiento de dichas campañas a nivel local y provincial, además de intentar comprobar el grado de cumplimiento de las mismas y si éstas fueron desarrolladas siguiendo patrones implantados a nivel nacional. Los datos obtenidos de las fuentes primarias se contextualizaron en el marco de estudios previos llevados a cabo en otras zonas geográficas españolas y también se pusieron en relación con las directrices dadas por los organismos nacionales e internacionales.

\section{LAS RECOMENDACIONES DE LAS AGENCIAS Y ORGANISMOS INTERNACIONALES SOBRE LOS PROGRAMAS DE VACUNACIÓN}

Desde 1953, la OMS incorporó la poliomielitis entre sus actividades y creó comités de expertos que elaboraron una serie de informes técnicos para orientar las acciones a emprender por los diferentes países y regiones.

En otro momento, tuvimos la ocasión de estudiar detalladamente el contenido y la secuencia de las recomendaciones de la OMS sobre las estrategias que un país debería adoptar para poner en marcha programas poblacionales de vacunación antipoliomielítica (Porras Gallo, Báguena Cervellera, Ballester Añón, 2010) y, también, las discusiones en las Conferencias Internacionales sobre Poliomielitis, en los Simposia de la Asociación Europea frente a la Poliomielitis y en los trabajos llevados a cabo por la $\mathrm{Na}$ tional Foundation for Infantile Paralysis (NFIP) (Ballester Añón, Porras Gallo, Báguena Cervellera, 2013). En estos estudios nos ocupamos también de la presencia española en dichos foros que, en líneas generales, tuvo un grado de implicación creciente, especialmente a partir de los años sesenta, con el grupo de epidemiólogos y virólogos de la Escuela Nacional de Sanidad.

El programa de vacunación parcial llevado a cabo por la Escuela Nacional de Puericultura en 1957, con vacuna Salk importada, se desarrolló con una clara falta de medios humanos y materiales e hizo inviable la determinación de anticuerpos. Más tarde, y recogiendo las indicaciones emanadas por dichos organismos internacionales, la puesta en marcha de la campaña masiva de vacunación de 1963-64 se desarrolló conforme a dichas indicaciones (Ballester Añón, Porras Gallo, 2009). Por lo demás, se insistía en la necesidad de vacunar poblaciones completas, por la existencia de portadores sanos, de establecer redes de laboratorios de virología y de mantener la cadena del frío para preservar la pureza de la vacuna (Porras Gallo, Báguena Cervellera, 2015).

\section{ETAPAS EN EL DESARROLLO DE LA VACUNACIÓN ANTIPOLIOMIELIITICA EN ALICANTE}

Las iniciativas preventivas llevadas a cabo en el espacio alicantino se desarrollaron en tres fases: los antecedentes sobre este proceso en el periodo previo a 1963; los años correspondientes a la primera campaña de vacunación oral antipolio y el periodo inmediatamente posterior 1963-65; y, finalmente, la década 1966-75, fecha, esta última, en la que se implantó el calendario vacunal en España.

\section{ANTECEDentes. El PeRIOdO PREVIO A LA CAMPAÑA NACIONAL DE VACUNACIÓN CONTRA LA POLIO DE 1963}

La relevancia que supuso el descubrimiento de la vacuna contra la poliomielitis y la primeras campañas de vacunación masiva en el extranjero, realizadas en 1955 , fueron recogidas en numerosas noticias publicadas en medios de comunicación nacionales (Ferrándiz, 1971; Porras Gallo y Báguena Cervellera, 2008).

En el V Symposium europeo sobre Poliomielitis, celebrado en Madrid en 1958, se abordó la implantación de la primera campaña de vacunación antipoliomielítica, realizada en España en el año 1957, en la que se aplicó la vacuna Salk, aproximadamente, a 5.000 niños. Esta campaña continuó en 1958, alcan- 
zando cifras de inmunización más elevadas (Laguna, 1958, pp. 87-88).

El principal motivo por el que ésta primera iniciativa de inmunización fue realizada a pequeña escala se encuentra en el escaso nivel de recursos económicos destinados a la adquisición de dosis de vacuna en cantidad suficiente, lo que influyó de forma notable en un inadecuado modelo de implantación financiado, en su mayoría, por fondos privados (Rui Pita y Rodríguez-Sánchez, 2008, pp. 331-334). A pesar de este panorama de escasez propio de un régimen franquista que en ese momento iniciaba una lenta recuperación de su imagen política a nivel internacional, las autoridades sanitarias llevaron a cabo un modelo de inmunizaciones dirigidas hacia grupos específicos de población como un nuevo ejemplo, dentro del conjunto de iniciativas propagandísticas, del interés por ofrecer una visión diferente a la realidad nacional.

El inicio de la aplicación en España de la vacuna Salk coincidió con una explosión epidémica sin precedentes (Rodríguez-Sánchez y Seco Calvo, 2009). Los años posteriores, 1958-59, superaron los dos mil casos, algo nunca sucedido en España, alcanzando en 1959 el máximo histórico. A pesar de disponer de una vacuna efectiva, y de haber comenzado campañas de vacunación, las cifras de casos declarados indicaban que la situación en el año 1961 era todavía muy preocupante (Nájera et al, 1975).

En el inicio de la década de 1960, distintos foros científicos y medios de comunicación comenzaron a prodigar las ventajas de la vacunación oral de Sabin frente a la vacuna de Salk. La facilidad en su administración, así como la mayor inmunidad ofrecida, favoreció que entre los años 1960-64 la mayoría de países occidentales, incluyendo EEUU, la utilizaran en sus campañas de inmunización.

Aunque la primera campaña masiva de vacunación oral antipoliomielítica no fue iniciada hasta 1963, a finales de 1961 España ya estaba preparada para llevarla a cabo a falta, tan sólo, de la decisión política y los recursos económicos necesarios. Pero esta autorización se retrasó casi dos años debido, entre otros factores, al conflicto entre instituciones -Instituto Nacional de Previsión (SOE), Dirección General de Sanidad (DGS) o Escuela Nacional de Sanidad (ENS)-, pertenecientes a Ministerios distintos, de Interior y de Trabajo, con presupuestos muy desiguales y con competencias solapadas en la organización de sus recursos. El conflicto sobre la asignación de acciones entre el SOE y la DGS se centró en la conveniencia de aplicar la vacuna Salk o la vacuna oral de Sabin. Además de los factores políticos y económicos que retrasaron el inicio de esta primera campaña existieron otros de tipo ideológico, que también influyeron en la elección de la vacuna (Tuells Hernández, 2008, pp. 321-324; Rodríguez Sánchez y Seco Calvo, 2009).

A pesar de la escasez de documentación existente relativa a este periodo cronológico, las fuentes archivísticas de tipo local y provincial contienen información relacionada con las primeras acciones de inmunización llevadas a cabo en la provincia de Alicante en el periodo previo a la campaña de vacunación oral de 1963. A lo largo de los años 1961-62 son cursadas solicitudes de dosis de vacuna antivariólica, antitífica y antipolio $^{1}$.

\section{De LA PRIMAVERA DE 1963, Y EL INICIO DE LA PRIMERA CAMPAÑA DE VACUNACIÓN ORAL ANTIPOLIOMIELÍTICA A 1965}

En enero de 1963, una orden ministerial ${ }^{2}$ sentó las bases de la primera Campaña Nacional de Vacunación Antipoliomielítica con vacuna oral (Porras Gallo y Báguena Cervellera, 2008; Rodríguez Sánchez y Seco Calvo, 2009). Esta primera iniciativa fue planteada en dos fases (noviembre-diciembre de 1963 y abril-mayo de 1964), en las que previamente se realizó una extensa campaña de divulgación propagandística en prensa, radio, NO-DO y televisión sobre las ventajas de la vacunación. El objetivo del régimen era intentar transmitir confianza sobre la efectividad de la vacuna.

A partir de ese momento aumentaron los esfuerzos de la DGS por hacer realidad el compromiso de vacunar de forma masiva a la población infantil de riesgo. La disminución de los casos observados tras la primera campaña antipoliomielítica demuestra una gran utilización de recursos económicos y humanos en número suficiente como para llevar la vacuna, por medio de unidades móviles, a todo el territorio español (Porras Gallo y Báguena Cervellera, 2015; Rodríguez Sánchez, 2015). La vacunación fue aplicada sobre población comprendida entre dos meses y siete años de edad (Nájera et al, 1975) alcanzando, según fuentes de archivo, un porcentaje superior al $95 \%$ en todas las provincias españolas.

A pesar del aparente éxito de esta iniciativa, resultados posteriores pusieron en duda esta afirmación, los 
casos de polio registrados y el insuficiente número de vacunaciones practicadas certificaron que la campaña de vacunación no alcanzó a todos los menores de 7 años (Valenciano, Mezquita, Pérez Gallargo, Gabriel y Galán, 1969; Porras Gallo y Báguena Cervellera, 2015).

De forma paralela a las iniciativas de ámbito nacional practicadas desde el inicio de 1963 , si nos centramos en el estudio de la provincia de Alicante, en un periodo inmediatamente anterior al inicio de la primera campaña oral, la Jefatura Provincial de Sanidad aceptó el ofrecimiento realizado por la DGS para el envío de vacuna a las localidades que la solicitaran.

Las fuentes archivísticas revisadas nos permiten tener acceso al constante flujo de documentación, escrita en forma de solicitudes por parte de los médicos titulares y jefes locales de sanidad de prácticamente todas las poblaciones de la provincia de Alicante, requiriendo dosis de vacuna antipolio. Algunas muestras de primeras acciones emprendidas son fechadas antes del inicio de la primera campaña oral de vacunación. Una prueba de esta interacción entre municipios y la Jefatura Provincial de Sanidad es el escrito cursado por la Inspección Municipal de Sanidad de la localidad de Ibi, con fecha 10 de enero de 1963 , en el que se confirman inmunizaciones inyectadas con la vacuna Salk a 41 niños de la localidad y se solicita un nuevo envío para aplicar una segunda vacuna, planificada para el 9 de febrero. La respuesta de este organismo, atendiendo a lo solicitado, se produjo el 6 de febrero de $1963^{3}$.

Posteriormente, desde abril de 1963, mediante escrito de la Sección de Epidemiología de la Jefatura Provincial de Sanidad a diferentes poblaciones de la provincia, se informa de la intención de iniciar una campaña de vacunación antipoliomielítica para la que, en consecuencia, serán enviadas las dosis de vacuna necesarias en cada caso. A partir de esa fecha, los escritos dirigidos a la Jefatura Provincial de Sanidad se suceden constantemente por parte de todas las localidades de la provincia de Alicante. Existe una abundante documentación en forma de solicitudes, firmadas por inspectores y jefes locales de sanidad, médicos titulares y alcaldes de la localidad - junto con respuestas emitidas por la Jefatura Provincial de Sanidad - requiriendo dosis de vacuna para emprender la campaña antipolio.

La elevada demanda de vacuna, representada por los requerimientos que hemos podido revisar, más de 150 escritos cursados entre los meses de abril y octubre de 1963 solicitando dosis de vacuna antipolio, no se relaciona con los recursos económicos ni organizativos existentes para llevar a cabo esta tarea. Son numerosos los ejemplos en los que el número de dosis solicitadas no coincide con las cantidades enviadas por la Jefatura Provincial. En estos casos se producen, de forma reiterada, nuevas peticiones ${ }^{4}$.

Teniendo en cuenta que la campaña de vacunación oral se implantó en noviembre de 1963, y que la documentación archivística de tipo local a la que hemos tenido acceso menciona la intención de la Jefatura Provincial de Alicante de iniciar una campaña de vacunación, programada para su inicio, el 2 de abril de 1963, ésta solo pudo ser llevada a cabo con la vacuna de virus inactivados de Salk, de forma previa a la primera campaña de vacunación Sabin.

Las carencias en el procedimiento y la deficiente gestión sanitaria es más evidente en poblaciones pequeñas y del interior de la provincia, donde se observa cómo las características rurales y las dificultades de desplazamiento al lugar, día y fecha convocada influyeron de forma notable en el no cumplimiento de las tasas de vacunación ${ }^{5}$. A estas dificultades habría que sumar el índice de analfabetismo existente y los prejuicios de la población hacia la vacunación de sus hijos debido a las noticias publicadas de casos de polio en niños vacunados (Porras Gallo, Ayarzagüena Sanz, De las Heras Salord, Báguena Cervellera, 2013; Porras Gallo y Báguena Cervellera, 2015). Otros obstáculos, derivados de la lectura de la correspondencia entre la jefatura local y provincial fueron la ausencia de un practicante que pudiera llevar a cabo las vacunaciones; la escasez de fichas empleadas en el control de las inmunizaciones; dificultades de criterios en el proceso de selección de los niños ante un número de vacunas insuficiente; la coexistencia de grupos de niños de primera inyección junto con otros grupos que requerían una segunda o tercera dosis; la afectación de niños con tos ferina o sarampión, culpable del escaso número de pretendientes el día de la convocatoria; y deficiencias organizativas y en la gestión de recursos, favorecidas por enfrentamiento entre instituciones como DGS y SOE que provocaba la coexistencia de niños vacunados frente a otros que no lo habían sido.

Sobre la documentación revisada, el número de vacunas solicitadas ofrece una idea de la importancia de los brotes. Además, escritos remitidos por algunas localidades dejan ver las carencias en el procedimien- 
to y en el número de dosis requeridas para vacunar a todos los niños menores de 7 años de edad ${ }^{6}$.

Por otro lado, no existen fuentes de archivo distintas a las de prensa local que nos ofrezcan información del procedimiento llevado a cabo en la campaña de vacunación masiva oral de Sabin, y responsable del significativo descenso en las cifras de afectados entre 1964 y 1965.

Es lógico pesar que, al igual que en un ámbito nacional, la campaña de vacunación oral tambien fuera dotada, a nivel local, con una mayor cantidad de recursos. En esta nueva oportunidad, las autoridades sanitarias replantearon estrategias que no fueron tenidas en cuenta en el pasado. Una de la diferencias estriba en que la campaña realizada con vacuna Salk estuvo caracterizada por una divulgación mediática mucho más moderada. Este hecho es patente por el conjunto de noticias publicadas en prensa Alicatina entre los años 1957-62 (43 noticias), frente al grupo de noticias publicadas entre los años 1963-65, (154 noticias).

Una posible singularidad en el procedimiento de vacunación llevado a cabo en Alicante durante 1963, frente a otros casos nacionales, que ofrece una idea de la falta de un nivel organizativo adecuado entre instituciones -SOE y DGS-, radica en el hecho que la campaña de vacunación Salk, a pesar de ser iniciada a escala nacional en el año 1957-58, fue implantada en Alicante con posterioridad, entre abril y octubre de 1963. Si tenemos en cuenta que las fuentes documentales certifican el inicio de la primera campaña de vacunación oral en noviembre de 1963, la continuidad en el tiempo de estos dos programas de inmunización podría dotar de características propias el caso de Alicante. Una posible consecuencia de esta cercania en las campañas es la hipótesis de si esta planificación pudo influir en la tasa de éxito de inmunización posterior $y$, en consecuencia, ser considerado uno de los factores que contribuyera a una menor afectación de la provincia de Alicante respecto al resto de provincias nacionales, especialmente llamativa para los años 1964-1971, según se desprende del estudio de los datos derivados del INE base Historia, al comparar los totales de Alicante capital y provincia con los totales del pais y de las capitales de provincia. Según el Instituto Nacional de Estadística, y teniendo en cuenta el posible sesgo en los datos analizados debido a las dificultades de control, detección y notificación de los casos observados, durante la década 1966-75, el comportamiento de las cifras de morbili- dad, tanto en Alicante como en la provincia, no sigue el patrón observado a escala nacional, siendo más positivas en el caso local.

\section{LA FASE FINAL HASTA LA IMPLANTACIÓN DEL CALENDARIO VACUNAL (1966-1975)}

A pesar del éxito de la campaña de vacunación masiva antipoliomielítica de 1963, publicaciones próximas a 1970 demostraron que la evolución de las tasas de incidencia de la enfermedad no se producía de la forma en la que lo hacían en países extranjeros.

Aunque las series temporales son incompletas, y a pesar del vacío existente en los años posteriores a 1963, la documentación relativa a las campañas de vacunación triple y antipoliomielítica, especialmente la correspondiente a 1972, sí incluyen en algunos casos expedientes completos e informes sobre el desarrollo de las mismas. Ésta, de carácter gratuito para todas las clases sociales, fue ordenada por la DGS e inspirada en las mismas normas y pautas de ejecución que las anteriores. En la provincia de Alicante fue desarrollada en dos fases (del 2 al 21 de octubre y del 13 de noviembre al 2 de diciembre $)^{7}$ y para ello fueron dispuestos un total de 5 equipos fijos de vacunación.

La primera vacunación, monovalente tipo I, debía ser realizada a todos niños mayores de 3 meses y menores de 3 años que con anterioridad no hubieran recibido dos dosis. La segunda vacunación, trivalente, era aplicada con un intervalo de 35 días. Una dosis de recuerdo, monovalente tipo I, era inyectada en todos los niños menores de 5 años que en campañas anteriores hubieran recibido las dos dosis de primovacunación. Finalmente, una cuarta dosis era aplicada en el ingreso en las escuelas a los niños ya vacunados.

Otros apartados recogían instrucciones sobre aspectos relevantes en su procedimiento como: la localización y llamamiento de los afectados; el parte y notificación semanal del número de niños vacunados; el registro de las incidencias que pudieran suceder; así como el informe de evolución al final de cada fase.

Finalmente, tras más de una década de vacunación, España destacaba de forma negativa como uno de los pocos países desarrollados en los que no se podía afirmar la desaparición virtual de la enfermedad. Así como el uso de la vacuna fue eficaz para combatir el 
brote epidémico en la campaña inicial, su utilización en la década posterior como herramienta para el control y posterior eliminación de la enfermedad no tuvo el efecto deseado. Nuevamente, entre las principales causas del fracaso se identifican aspectos como la negligencia de los padres; la pauta vacunal, establecida en dos cortos periodos del año; la implantación de la vacunación en los centros preventivos en lugar de en los centros asistenciales, pues esta doble vía constituía un obstáculo para lograr la plena vacunación; y el reducido grupo de edad sobre el que se aplicó vacunación.

El diferente comportamiento de los casos observados, frente a otros países, indicaban que el sistema de vacunación en España no fue adecuado. Una prueba que demuestra la ineficacia de las campañas de vacunación realizadas hasta la fecha es que en el año 1975 un total de 20 capitales de provincia presentaban tasas de mortalidad superior a cero, siendo la morbilidad en España, en ese año, la más elevada de toda la cuenca Mediterránea. El punto más bajo a nivel nacional (62 casos en 1965) no pudo ser mejorado hasta 1976, con 41 casos registrados (Nájera et al, 1975). Fue a partir de ese momento, justificado por la implantación del calendario vacunal, cuando España comenzó a vislumbrar la posibilidad de erradicación de la enfermedad, aunque ésta no se conseguiría de forma completa hasta 1988.

\section{LA VACUNACIÓN Y CAMPAÑAS EN LA PRENSA}

El análisis de la prensa local permite identificar la repercusión de la poliomielitis y de sus medidas preventivas durante las tres etapas cronológicas propuestas. Un ejemplo lo constituye el estudio realizado sobre las noticias del Diario Información de la provincia de Alicante, en el que se registraron un total de 343 noticias relacionadas con la poliomielitis, sus medidas terapéuticas y su vacuna entre 1950 y 1975. Dicho periódico constituía la fuente de información provincial más representativa, tanto en los años de estudio como en la actualidad, y formaba parte de un modelo de prensa donde el poder político y la palabra convivían bajo un estricto modelo totalitarista.

\section{ANTECEDENTES. El PERIOdO PREVIO A LA CAMPAÑa NACIONAL DE VACUNACIÓN CONTRA LA POLIO DE 1963}

A partir de 1950, un escaso número de noticias publicadas relatan la preocupación mundial por el comportamiento de la enfermedad. Los años 1955 y 1956 muestran un aumento importante en el número de noticias, con un total de 20 titulares, respectivamente, de ámbito internacional y nacional.

Un aspecto característico de este periodo es la escasa repercusión de las noticias relacionadas con el comportamiento de las epidemias en España, a diferencia del notable eco de las novedades en el extranjero, especialmente en EEUU. José Antonio Palanca, director general de sanidad, niega la existencia de epidemias en España y califica como "reducidísimos" los casos declarados hasta la fecha. Esta postura es también defendida por profesionales de reconocido prestigio como el dr. Bosch Marín, aunque se destaca cierta preocupación por la posibilidad de que esta situación pueda empeorar en el futuro.

La vacuna Salk y su eficacia son noticias que sucesivamente se repiten a lo largo de estos años. Los avances de científicos y de los laboratorios encargados de fabricarla ocupan gran parte de las noticias. Otro tipo de titulares, éstos con un objetivo más propagandístico, destacan la posición de España dentro del grupo de países preparados para llevar a cabo las primeras campañas de vacunación.

La primera noticia sobre vacunaciones en España se produce el 29 de febrero de 1956, y su titular recoge la "autorización de la vacunación antipolio en España". En este año, el 18 de agosto, se publica la primera noticia que constata la declaración de casos a nivel nacional, un total de 3 en una misma familia de Burgos. Entendemos que este hecho denota un punto de inflexión en la colección de noticias en prensa, consecuencia del aumento de la preocupación nacional, ya que la serie de 1956 finaliza con la publicación, por primera vez, de información sobre cuidados generales, profilaxis, pautas de actuación y diagnóstico diferencial de la parálisis infantil.

En 1957 se refleja la posibilidad de obtener una nueva vacuna, de tipo oral, con mayores ventajas de inmunidad y una protección más duradera. Un año más tarde, en 1958, el discurso del dr. Bosch Marín denota un cambio en las premisas expuestas con an- 
terioridad, mostrándose partidario de las vacunaciones.

Justificado por la influencia de esta nueva corriente, se observan diferencias en el tratamiento de las noticias por parte de la prensa local. Los titulares se dirigen ahora hacia aspectos como: el esfuerzo por conseguir una vacuna más barata, el sentido de la prevención y el momento más adecuado para hacerlo, la necesidad de aplicarla a los menores de 15 años, así como a la importancia de las medidas de rehabilitación.

A nivel local, las directrices propagandísticas relacionadas con las primeras iniciativas de inmunización Salk se dejan ver en forma de convocatorias provinciales de vacunación, aunque en un periodo de tiempo posterior a $1957^{8}$.

Con la excepción de 1959, año que destaca con un total de 10 noticias, los años posteriores, hasta 1963, se caracterizan por una ausencia de noticias relacionadas con la polio - únicamente 14 reseñas fueron publicadas entre los años 1960 y 1962, las cifras más bajas de toda la serie de años comprendida entre 1955 y 1968-. La moderación mediática sobre las acciones relacionadas con los discretos programas de vacunación Salk es manifiesta cuando se compara con la campaña oral de Sabin. Este control informativo cambiará notablemente a partir de 1963, cuando el país cuenta con los suficientes recursos económicos y humanos para hacer efectiva una vacunación masiva9.

La lectura de las notas de prensa publicadas en el inicio de la década de 1960 denota temáticas superficiales nada relacionadas con la situación vivida en el país en cuanto a cifras de afectados. En los años posteriores, titulares de victoria frente a la poliomielitis, consecuencia de los hitos logrados en el campo experimental de la vacuna oral, se siguen produciendo de forma solapada con aspectos relacionados con la vacuna desarrollada por Salk.

\section{DE LA PRIMAVERA DE 1963, Y EL INICIO DE LA PRIMERA CAMPAÑA DE VACUNACIÓN ORAL ANTIPOLIOMIELÍTICA A 1965}

A partir del año 1963, con los preparativos dispuestos para el inicio de la campaña masiva de vacunación oral, la presencia de noticias aumenta de forma notable siguiendo un guion pautado por las autoridades sanitarias para concienciar a la población de la importancia de ser vacunado. El papel desarrollado por los medios de comunicación formaba parte de las acciones planificadas para sensibilizar a la población de la necesidad de vacunar a sus hijos. A partir de ese momento, las cifras de afectados son publicadas en prensa junto con amplios titulares que anuncian el inicio de una nueva campaña de vacunación, ofreciendo una imagen de control y tranquilidad a la población al disponer los recursos necesarios para hacer frente, de igual modo que otros países, al avance de la polio.

Otras acciones contempladas en este planteamiento de difusión mediática, con influencia sobre la opinión pública, fueron las declaraciones de científicos de reconocido prestigio que visitaron España durante ese periodo. Nos referimos a las conferencias realizadas en Madrid por Albert Sabin o Pierre Lépine, entre otros, en el año 1963, con el objetivo de proyectar confianza sobre la efectividad de la vacuna oral.

El mayor protagonismo de noticias, ocupando un destacado lugar en la prensa alicantina, son las referencias a las dos campañas de vacunación nacional desarrolladas durante el año de 1963. En la primera, entre enero y junio, auspiciada por SOE, con vacuna Salk y con un total de 16 crónicas, la utilización de vacuna de primera calidad y su carácter voluntario para niños menores de siete años aparece en lugar destacado. La escasez de recursos se justifica en prensa anunciando que la vacunación será dirigida hacia los grupos de población más desfavorecidos. La segunda campaña, posterior a la de Salk, realizada con vacuna oral e iniciada entre noviembre y diciembre de ese mismo año, se refleja en prensa con un total de 37 noticias.

A diferencia de lo ocurrido en la campaña realizada con vacuna Salk, la prensa destaca las palabras del director general de sanidad, García Orcoyen, y del director de la campaña, Pérez Gallardo, sobre las ventajas de la vacuna oral, el esfuerzo realizado por la administración y la enorme cantidad de profesionales implicados. El resto de sus dos fases, iniciadas a partir del siguiente año, y las primeras estimaciones, extraordinariamente positivas ${ }^{10}$, son noticias destacadas en 1964.

La primera noticia que proclama el inicio de esta campaña en la provincia de Alicante, encabezada con el titular "Comenzó la vacunación antipolio en la pro- 
vincia", localiza esta iniciativa en la localidad de San Juan. El mismo titular anuncia que "Los niños de Alicante serán vacunados del 9 al 14 próximo". Las cifras de vacunados, tal como recoge la noticia, alcanzan numerosas poblaciones, al tiempo que se proyecta una imagen de actuación mucho más organizada. Otro ejemplo de eficacia es la noticia reflejada en prensa, el 5 de diciembre de 1963, sobre la respuesta de la población censada en la localidad de Crevillente, en la que fueron vacunados un total de 3.000 niños en un periodo cercano a tres horas. La reseña agradece la labor divulgativa prestada a aquellos por los que sin su ayuda no hubiera sido posible alcanzar esta tasa de éxito.

La prensa del día 8 de diciembre recoge la noticia del inicio programado de las vacunaciones en Alicante entre el 9 y 14 de diciembre. Se estima que un total de 15.000 niños censados con edades comprendidas entre los dos meses y 7 años de edad fueron vacunados en alguno de los puestos fijos y equipos móviles estratégicamente dispuestos en la zona. Durante 1964 se suceden constantemente noticias con cifras de vacunaciones, tanto en la provincia como en la capital.

Pero serán los excelentes resultados, amparados por el descenso de la mortalidad y la morbilidad de la enfermedad tras esta campaña, y el inicio de la inmunización con polio oral trivalente junto con la vacunación frente a la difteria, tétanos y tos ferina (DTP) ${ }^{11}$, las referencias que obtuvieron una mayor representación durante 1965 . A partir de entonces se implantaron de forma continuada las campañas de vacunación frente a la polio y DTP. En este año se suceden numerosos recordatorios de vacunación para los niños con edades comprendidas entre 3 y 12 meses, junto con noticias de cifras de inmunizaciones. Los mensajes de victoria sobre la enfermedad se repiten, advirtiendo la importancia de la continuidad en el proceso de vacunaciones para no dejar opción al virus. Las campañas de vacunación contra la difteria, tos ferina y el tétanos ocupan ahora un mayor protagonismo en prensa y son calificados como "tres enemigos de la paz de los niños". A partir de 1966 el grupo de noticias se centra, fundamentalmente, en datos sobre la evolución de las campañas y recordatorios sobre las fechas, lugares de realización y horarios de vacunaciones, poniendo de manifiesto la importancia concedida desde el ámbito médico y político a la concienciación de los padres y a la importancia de completar la pauta vacunal.

\section{LA FASE FINAL HASTA LA IMPLANTACIÓN DEL CALENDARIO VACUNAL (1966-1975)}

Es a partir de 1966 cuando la progresiva reducción en el volumen de noticias publicadas se correlaciona con la reducción de las cifras de casos observados, tanto a nivel nacional (Porras Gallo, Báguena Cervellera, 2015) como a nivel local, siendo mucho más positivas en este último escenario. No obstante, esta situación de relajación social, favorecida por la escasa actividad propagandística y por un sentimiento triunfalista ante la erradicación, intentó corregirse más tarde, a partir de 1970-71, dadas las elevadas cifras de morbilidad aún existentes y el repunte de nuevos casos observados entre 1972 y 1975 a nivel provincial.

Una nueva tendencia de progresivo aumento en el número de noticias publicadas se produjo entre los años 1970 y 1975, coincidiendo con el aumento de casos en la provincia de Alicante. Resulta llamativo que, a pesar del aumento de los casos en la provincia de Alicante entre 1972-75, la prensa local sólo publicara una noticia sobre el tema. Ésta se produjo el 14 de octubre de 1972, con la declaración de 15 nuevos casos en Alicante, Elche, Catral y Callosa del Segura. El resto de noticias, hasta el final de la serie de estudio, son recordatorios de campañas de vacunación y noticias relacionadas con la sensibilización social de las discapacidades producidas por la enfermedad.

\section{NARRATIVAS VACUNALES}

Las estructuras narrativas permiten organizar y dar significado a las experiencias (Turner y Bruner, 1986). La cincuentena de testimonios recogidos mediante la técnica de la historia oral ${ }^{12}$ nos permiten completar desde el otro lado, el de las personas afectadas, el análisis del problema estudiado. Como comentamos arriba, y a diferencia de lo que constituye una herramienta de análisis usual en la salud pública actual (Abeysinghe, 2015), es mucho más infrecuente en la historiografía médica donde, sin embargo, hay un estimable número de estudios sobre experiencias de enfermedad en personas que contrajeron la enfermedad. Además, aunque en algún caso se aborda el tema de las vacunas, éste está integrado en el marco más amplio de las historias de vida ${ }^{13} \mathrm{o}$, como recientemente se ha abordado, en temas cercanos como el uso de las narrativas en los primeros ensayos clínicos con gammaglobulina (Mawdsley, 2016). 
Todas las personas entrevistadas contrajeron la enfermedad en un abanico amplio de fechas, entre 1934 y 1974. La mayor parte de ellas (70\%), entre 1955 y 1963 , coincidentes con el periodo presencia de brotes epidémicos en la provincia alicantina. Todas ellas padecen, en mayor o menor medida, secuelas de la enfermedad y en algún caso, Síndrome postpolio. La mayor parte de ellas (66\%) no fueron vacunadas, otras lo desconocen (9\%) y sí lo recuerda, el resto.

A través de aspectos como los conocimientos sobre las vacunas y campañas de vacunación, sentimientos sobre su propia experiencia personal y percepción de las causas del fracaso de las inmunizaciones, en algunos casos, las respuestas no son uniformes y revelan cómo las diferentes trayectorias biográficas modulan los significados que los informantes dan a estas cuestiones.

El conocimiento sobre las vacunas antipoliomielíticas o el tipo de vacuna que se suministraba antes de 1975 , es escaso. Hasta el punto que no es infrecuente la idea de que antes de que aparecieran los brotes de los años cincuenta, ni siquiera se conocía la enfermedad. En cuanto al tipo de vacuna, de forma esporádica aparecen los nombres de Salk o Sabin, pero prácticamente todos los informantes refieren las gotas en el terrón de azúcar.

Una mezcla de sentimientos de rebeldía y de resignación se produce en aquellos para los que la vacuna llegó demasiado tarde, y ello es especialmente marcado en aquellos que contrajeron la enfermedad poco antes de que se iniciaran las campañas sobre las que hay disparidad y baile de fechas: "No existía la vacuna. La vacunación comenzó poco después de que yo cayera enfermo. El diagnóstico coincidió con las primeras vacunaciones"; "No fui vacunado porque aún no había salido la vacuna al mercado. Salió, al final, en 1961 en algunos sitios y a partir de 1968 se hizo más extensiva"; en otro caso, "No llegué a vacunarme porque estaba con una bronquitis y no se podía".

Y una clara sensación de agravio hacia los responsables de las políticas sanitarias y a los médicos, con la sensación de vivir en un país atrasado. "En aquella época no existía la vacuna. Cuando me dio a mí (la enfermedad) en 1956, fue cuando en estados Unidos la sacaron. Claro, aquí en España, ni se hablaba de ello"; "Fui vacunado, probablemente, a destiempo. La vacuna estaba entre dos aguas. Se suponía que la tenía el gobierno, pero no la había facilitado todavía". "Sí, fui vacunado pero la vacuna estaba todavía en periodo de experimentación y en aquellos momentos no eran fiables al cien por cien. Fui vacunado antes de las campañas y cuando me diagnosticaron, me volvieron a vacunar para tratar de solucionar el problema, pero ya era tarde"; y una frase común en los polios de aquí y de otros ámbitos geográficos: "Nací demasiado pronto" ("born too early"); "Para mí la vacuna llegó tarde, cuando empezaron a vacunar, ya era tarde para mí".

Pero no solo la fatalidad, también la falta de acceso: “No, no me vacunaron. Contraje la enfermedad en noviembre de 1962. Tuve la desgracia de que en Alicante llevaban 8-9 meses vacunando, pero a Novelda, no llegó"; "No se vacunaba en pueblos tan pequeños como Dolores y menos, si se trataba de una familia pobre". El tema de la pobreza como elemento que explicaba el no haber sido vacunado se repite en otros informantes. "La gente más humilde no se vacunaba"; "No fui vacunado (en Orihuela) porque faltaban vacunas. No había para todos", "no en todos los pueblos se vacunaba, había que llevar a los niños a la capital".

$\mathrm{Y}$, junto a ello, otros factores dependientes de lo que consideran una mala práctica médica: "Sí que fui vacunada en 1969. De hecho, por una negligencia médica la propia vacuna me ocasionó la enfermedad cuando yo estaba bien. Aquella misma noche me subió la fiebre y me fallaban las piernas"; "La vacuna se llamaba Sabin. Creó un cierto rechazo porque los primeros que se vacunaron quedaron polios". La ignorancia o la actitud en contra de las familias. "No fui vacunado por desconocimiento. Si había vacunas mi familia no lo sabía"; "No fui vacunado porque mis padres tenían miedo a los efectos de la vacuna".

\section{CONCLUSIONES}

Aunque la campaña de vacunación Salk fue iniciada a nivel nacional en 1957, en el caso de Alicante ésta se desarrolló con cierto retraso y su implementación (desde abril hasta octubre de 1963) se produjo en un periodo inmediatamente anterior a la primera campaña de vacunación de masiva oral Sabin. Quizás, esta continuidad en los programas de vacunación pudo influir en la tasa de éxito de inmunización posterior y ser considerado uno de los factores que contribuyera a una menor afectación de la provincia respecto al resto de provincias nacionales, especialmente llamativa por el diferente comportamiento nacional y local, entre los años 1966-1971.

Existe una abundante documentación, en forma de solicitudes, requiriendo dosis de vacuna a la jefatura 
provincial de sanidad. Gracias a ella hemos podido reconstruir, en parte, la práctica de las vacunaciones en el caso de Alicante en el periodo previo a la primera gran campaña con vacuna oral. Las cantidades solicitadas ofrecen una idea de la severidad del periodo epidémico y las noticias publicadas en la prensa alicantina recogen con fidelidad la situación vivida.

El protagonismo de la prensa en la campaña de vacunación oral de Sabin, junto con un mayor despliegue de medios, fueron factores decisivos en la reducción de casos observados en los años posteriores. Los esfuerzos realizados por las instituciones y por aquellos que tomaron parte activa en su implantación, la facilidad de la vía de administración de la vacuna, su carácter gratuito y una adecuada publicidad sobre la importancia de la vacunación favorecieron el éxito. A partir de 1966, la relajación de las medidas disuasorias, junto con la ineficacia de las campañas de vacunación en el control de la enfermedad, favoreció un repunte de casos entre 1966-75. Este incremento también llevó asociado un mayor protagonismo de la poliomielitis en prensa.

Aunque del estudio comparado con otras capitales de provincia y con el territorio nacional se desprende que Alicante no fue una capital de provincia especialmente afectada por la enfermedad, el comportamiento se muestra de forma análoga hasta 1965. En la década siguiente, desde 1966 a 1975, las cifras de morbilidad se comportan de forma diferente. En una primera fase, 1966-71, tanto la capital como el resto de la provincia presentan cifras de morbilidad inferiores a las correspondientes a nivel nacional. Es posteriormente, entre los años 1972-75, cuando se produce un cambio en la tendencia de los casos registrados, a favor de la provincia, frente a una inercia progresiva hacia la erradicación de la enfermedad, constatable tanto a nivel nacional como en Alicante capital.

A pesar de estas diferencias, los resultados revelan una cierta concordancia entre lo observado por otros autores (Porras Gallo y Báguena Cervellera, 2013; Porras Gallo y Báguena Cervellera, 2015; Rodríguez Sánchez y Seco Calvo, 2009; Rodríguez Sánchez, 2015) y lo reflejado en nuestro estudio a nivel local, y confirman la ineficacia en la implementación de las medidas de vacunación (Tuells Hernández, 2008). Por otro lado, la prensa local y la documentación archivística revisada permite confirmar cómo los escasos recursos para llevar a cabo los programas de inmunización, junto con carencias en el procedimiento, influyeron de manera determinante en el no cumplimiento de las tasas de vacunación.

La conclusión más evidente, a la vista de resultados posteriores derivados de las acciones llevadas a cabo tras la campaña de 1963, es que la ineficacia en el control de las medidas adoptadas a escala nacional fue también trasladada a un ámbito local y justifica los esfuerzos de las autoridades sanitarias hacia una mayor disposición de recursos enfocados a la contención de la enfermedad en núcleos rurales, donde la provincia de Alicante ocupaba, en 1975 , el séptimo lugar dentro del grupo de las 28 provincias con algún caso declarado.

En este marco general, las narrativas vacunales nos aportan información complementaria de cómo la población afectada vivió la experiencia de las vacunas y nos reflejan, además, la cara oculta de las vivencias personales en una población que, por un motivo $u$ otro de los que hemos analizado, no se vieron favorecidas por la novedad de estas nuevas tecnologías al servicio de la salud pública.

\section{AGRADECIMIENTOS}

Los autores agradecen a Dạ Susana Llorens Ortuño, responsable del Archivo Municipal de Alicante su inestimable ayuda, al jefe del Servicio de Rehabilitación del Hospital General de Alicante, JM. Climent Barberá y al profesor de Historia del Periodismo de la Universidad Miguel Hernández, Miguel Ors Montenegro Trabajo desarrollado en el marco del proyecto coordinado de I+D, ref. HAR2012-39655-C04-01 (Ministerio de Economía y Competitividad) y del Programa Prometeo de la Generalitat Valenciana (Prometeo fase II / 015). 


\section{NOTAS}

1 Archivo Municipal de Alicante - Signatura 240. Registro de entrada de documentos ( 2 enero 1960 a 1 de junio de 1963).

2 Orden de 26 de enero de 1963 de Ministerio de la Gobernación (B.O.E., 30-1-1963, no 26, pp. 1649-50).

3 Una nueva solicitud, correspondiente a la tercera fase de vacunación de estos 41 niños, fue remitida a la Jefatura Provincial el 5 de marzo de 1963, aunque la respuesta confirmatoria del envío, fechada el 11 de marzo, no responde a la cuestión planteada por el Jefe Local de Sanidad de lbi sobre la posibilidad de vacunar a todos los niños censados menores de 7 años de edad (un total de 383).

4 Sirva de ejemplo el escrito dirigido por D. Alfredo Garrido García, médico titular de Pinoso, al Jefe Provincial de Sanidad (04/04/1963) solicitando un total de 250 dosis de vacuna y la posterior comunicación, nuevamente, de D. Alfredo Garrido García certificando el envío de tan solo 90 dosis. Archivo Municipal de Alicante.

5 Escrito del jefe local de sanidad de Castalla, el 26 de abril de 1963 , dirigido a la jefatura provincial.

6 Un ejemplo es el caso de Ibi, con un total de 383 dosis solicitadas el 4 de abril, frente una cifra de 180 dosis recibidas el 9 de abril de 1963. Archivo Municipal de Alicante.

7 Escrito remitido por la Sección de Epidemiología de la Jefatura Provincial de Sanidad, de 29 de septiembre de 1972, a la Sra. Delegada Provincial de la Sección Femenina, anunciando la Campaña de Vacunación Triple-Antipoliomielitica. Archivo Municipal de Alicante.

8 El 3 de mayo de 1960, en forma de nota de la alcaldía, se presenta una de las primeras convocatorias de las que tenemos constancia en la prensa local. Este formato de

\section{BIBLIOGRAFÍA}

Abeysinghe, Sudeepa (2015), "Vaccine narratives and Public Health. Investigating criticism of $\mathrm{H} 1 \mathrm{~N} 1$ pandemic vaccination", PLoS Curr., Feb 25. Edition 1. doi: 10.1371/currents. outbreaks.17b6007099e92486483872ff39ede178.

Aitken, Sally; D’Orazio, Helen; Valin, Stewart (2004), Walking fingers. The story of polio and those who lived in it. Ottawa, Vehicule Press.

Ballester Añón, Rosa; Porras Gallo, María Isabel (2009), “EI significado histórico de las encuestas de seroprevalencia como tecnología de laboratorio aplicada a las campañas de inmunización”, Asclepio, LXI, pp. 55-80.

Ballester Añón, Rosa; Porras Gallo, María Isabel (2012), “Dossier: Políticas y respuestas sociales contra la poliomielitis en Europa", Dynamis, 32, pp. 273-414.

Ballester Añón, Rosa; Bernanebeu Mestre, José; Castejon Ortega, Ramón; Perdiguero Gil, Enrique (eds.) (2012), Salud y citación será utilizado en posteriores ocasiones con gran frecuencia.

9 Algunos autores ven en este cambio de proceder un intento de legitimización del régimen, de proyección de imagen internacional como una nación preparada, como medio de conseguir su aceptación final en organismos de tipo internacional (Rui Pita y Rodríguez-Sánchez, 2008).

10 El 24 de marzo de 1964 la prensa alicantina destaca el proceso de vacunación en la provincia de Alicante como ejemplo de eficiencia -un total de 112.131 niños vacunados de los que 20.000 pertenecen a la capital- en el que, hasta la fecha, según la prensa, no se ha notificado ningún caso de polio.

11 Este momento es señalado por Fernando Ruiz Falcó, director de la Escuela Nacional de Sanidad, como el inicio de "una cultura de vacunas en España". (Tuells Hernández, 2008).

12 Se entrevistaron 51 informantes (33 varones y 18 mujeres) que tienen la doble peculiaridad de residir en la provincia de Alicante y haber padecido la polio y sus secuelas. Las entrevistas se realizaron en el Servicio de Rehabilitación del Hospital General de Alicante y en domicilios particulares, en un periodo de tiempo dilatado, entre 2010 y 2015.

13 Exclusivamente para el caso de la polio en España, y con contenidos de mayor amplitud que las experiencias vacunales stricto sensu, son de lectura obligada los trabajos: (Rodríguez Sánchez y Seco Calvo, 2004; Rui Pita, Rodríguez Sánchez, 2008; De las Heras, Porras Gallo, Báguena Cervellera, 2013; Rodríguez Sánchez, Ballester Añón, Porras Gallo y Báguena Cervellera, 2013). En el contexto internacional, son de referencia obligada: (Aitken, D'Orazio, Valin, 2004; Gould, 1995; Sass, Gottfried y Sorem 1996). En el aspecto más amplio de las discapacidades: (Humphries y Gordon, 1992).

enfermedad en la sociedad alicantina contemporánea. Alicante, Instituto de Cultura Juan Gil Albert.

Ballester Añón, Rosa; Porras Gallo, María Isabel; Báguena Cervellera, María José (2013), “La respuesta de las agencias internacionales (NFIP, OMS, AEP) al problema de la poliomielitis". En: Porras Gallo, María Isabel; Ayarzagüena Sanz, Mariano; De las Heras Salord, Jaime; Báguena Cervellera, María José (2013): El drama de la polio. Un problema social y familiar en la España franquista, Madrid. Libros de La Catarata. pp. 73-93

De las Heras, Jaime; Porras Gallo, María Isabel; Báguena Cervellera, María José (2013), “El drama de las víctimas de la poliomielitis visto por la prensa, por ellas mismas y por sus contemporáneos". En: Porras Gallo, María Isabel; Ayarzagüena Sanz, Mariano; De las Heras Salord, Jaime; Báguena Cervellera, María José (eds.), El drama de la polio. Un problema social y familiar en la España franquista, Madrid. Libros de La Catarata. pp. 170-192. 
Ferrándiz, Vicente (1971), Exposición clara y convincente de las peculiaridades de la parálisis infantil. Poliomielitis. Barcelona, Cedel.

Gould, Tony (1995), A summer plague: Polio and its survivors, London, Yale University Press.

Humphries, Stephen, Gordon, Pamela (1992). Out of the sight. The experience of disability. 1900-1950. Plymouth, Northcote House ed.

Laguna, Carlos (1958), "La vacunación contra la poliomielitis en España". En: Bosch Marín, Juan (dir.), Aportación Española al V Symposium europeo sobre Poliomielitis. Madrid, Servicios de Protección Maternal e Infantil, Ministerio de la Gobernación, Dirección General de Sanidad, pp. 87-88.

Martínez Navarro, Ferrán (2013), "Los estudios epidemiológicos sobre la poliomielitis en España antes de la vacunación", Rev. Esp. Salud Pública, 87(5), pp. 429-441.

Mawdsley, Stephen (2016), Selling science: polio and the promise of gamma globulin. New Brunswick, Rutgers UP.

Nájera Morrondo, Rafael (2013), "La última fase: la eliminación”, Rev. Esp. Salud Pública, 87(5), pp. 461-469.

Nájera, Enrique; Llácer, Ángel; Valenciano, Luis; Salmerón, Enrique; Martínez Navarro, Ferrán; Mezquita, Manuel; Pérez Gallardo, Florencio (1975), “Análisis epidemiológico de la situación actual de la poliomielitis en España", Revista de Sanidad e Higiene Pública, 49 (10), pp. 953-1025.

Ortiz Gómez, Teresa; Olagüe de Ros, Guillermo; Rodríguez Ocaña, Esteban; Menéndez Navarro, Alfredo; Gil-García, Eugenia; Luna Maldonado, Aurelio; Sevilla Olmedo, Ma Teresa; Gómez-Núñez, Antonio J. (eds.) (2008), La experiencia de enfermar en perspectiva histórica. XIV Congreso de Historia de la Medicina, Granada, Universidad de Granada.

Porras Gallo, María Isabel; Báguena Cervellera, María José (2008), "La poliomielitis en la España franquista a través de la prensa general (1940-1975)". En: Ortiz Gómez, Teresa et al. (eds.), La experiencia de enfermar en perspectiva histórica. XIV Congreso de Historia de la Medicina, Granada, Universidad de Granada.

Porras Gallo, María Isabel; Báguena Cervellera, María José (2015), "El conocimiento sobre la realidad de las campañas de vacunación contra la polio: su cobertura, su seguimiento, la percepción de la población sobre su eficacia y/o peligrosidad y el reflejo en la prensa diaria". En: Perdiguero Gil, Enrique (ed.), Política, salud y enfermedad en España: entre el desarrollismo y la Transición democrática. Elche, Universidad Miguel Hernández. Editorial digital UMH.

Porras Gallo, María Isabel; Ayarzagüena Sanz, Mariano; De las Heras Salord, Jaime; Báguena Cervellera, María José (2013), El drama de la polio. Un problema social y familiar en la España franquista, Madrid. Libros de La Catarata.

Porras Gallo, María Isabel; Báguena Cervellera, María José; Ballester Añón, Rosa (2010), "Spain and the international conferences on polio, 1940's-1960's", Dynamis, 30, pp. 117-144.
Rodríguez Sánchez, Juan Antonio (2015), “Del control a la erradicación. Salud Pública y atención primaria en la lucha contra la poliomielitis en España (1963-1988)". En: Perdiguero Gil, Enrique (ed.), Política, salud y enfermedad en España: entre el desarrollismo y la Transición democrática. Elche, Universidad Miguel Hernández. Editorial digital UMH.

Rodríguez Sánchez, Juan Antonio; Seco Calvo, Jesus (2004), “La perspectiva del paciente: memoria e historia de la poliomielitis en España". En: Ortiz Gómez, Teresa et al. (eds.), La experiencia de enfermar en perspectiva histórica. XIV Congreso de Historia de la Medicina, Granada, Universidad de Granada, pp. 341-343.

Rodríguez Sánchez, Juan Antonio; Seco Calvo, Jesús (2009), "Las campañas de vacunación contra la poliomielitis en España en 1963”, Asclepio, 61(1), pp. 81-116.

Rodríguez Sánchez, Juan Antonio; Ballester Añón, Rosa; Porras Gallo, María Isabel; Báguena Cervellera, María José (2013), "La experiencia de la poliomielitis desde la perspectiva de género". En: Porras Gallo, María Isabel; Ayarzagüena Sanz, Mariano; De las Heras Salord, Jaime; Báguena Cervellera, María José (eds.), El drama de la polio. Un problema social y familiar en la España franquista, Madrid. Libros de La Catarata. Pp. 211-232.

Rui Pita, Joao; Rodríguez Sánchez, Juan Antonio (2008), “Actitudes ante la polio en España y Portugal: estudio comparativo". En: Ortiz Gómez, Teresa et al. (eds.), La experiencia de enfermar en perspectiva histórica. XIV Congreso de Historia de la Medicina, Granada, Universidad de Granada, pp. 331-334.

Sass, Edmund; Gottfried George; Sorem Anthony (1996), Polio's Legacy: an oral history. Lanham, University Press of America.

Smallmann-Raynor, Matthew; Cliff, Andrew (eds.) (2006), Mainland Europe. Poliomyelitis. A world geography. Emergence to eradication. Oxford, Oxford U.P.

Toledo Marhuenda, José Vicente (2013), La poliomielitis en España (1880-1970) y su impacto sobre el desarrollo de las técnicas de fisioterapia. Elche, Universidad Miguel Hernández. Editorial digital UMH.

Tuells Hernández, José (2008), “Los testimonios de los expertos y su participación en las primeras campañas de vacunación antipoliomielítica en España". En: Ortiz Gómez, Teresa et al. (eds.), La experiencia de enfermar en perspectiva histórica. XIV Congreso de Historia de la Medicina, Granada, Universidad de Granada, pp. 321-324.

Turner, Victor; Bruner, Edward (1986), The Anthropology of experience with an epilogue by Clifford Geertz. Urbana \& Chicago, University of Illinois Press.

Valenciano, Luis; Mezquita, Manuel; Pérez Gallardo, Florencio; Gabriel y Galán, Jesús (1969), “Estudio epidemiológico y virológico de la poliomielitis en España durante el quinquenio 1964-1968", Revista de Sanidad e Higiene Pública, 43 (7-8), pp. 517-564. 